\title{
Using Neural Networks to Identify Atoms in HRTEM Images
}

Jakob Schiøtz ${ }^{1}$, Jacob Madsen ${ }^{1}$, Bjarke J. G. Østergaard ${ }^{1}$, Anders S. Dreisig ${ }^{1}$, Pei Liu ${ }^{2}$, Stig Helveg ${ }^{3}$, Ole Winther ${ }^{4}$, Jens Kling ${ }^{2}$, Jakob Birkedal Wagner ${ }^{2}$ and Thomas Willum Hansen ${ }^{2}$

1. DTU Physics, Technical University of Denmark, Kgs. Lyngby, Denmark.

2. DTU Nanolab, Technical University of Denmark, Kgs. Lyngby, Denmark.

3. Haldor Topsøe A/S, Kgs. Lyngby, Denmark.

4. DTU Compute, Technical University of Denmark, Kgs. Lyngby, Denmark.

We have developed a methodology, where deep convolutional neural networks can be used to identify local structures in high resolution transmission electron microscopy (HRTEM) images. The methods are available as Python modules under an open source license [1].

While conventional image processing and peak finding algorithms can be used to pinpoint the position of the atoms (within the uncertainty due to imaging effects) [2], the fact that contrast varies from image to image, and sometimes even inverts within a single micrograph makes such methods difficult to use. A neural network can act as an advanced filter, giving a clean signal for peak detection (Figure 1).

In most cases, using machine learning (ML) for image analysis is limited by the availability of highquality pre-analyzed images that can be used for training the ML methods; acquiring the training data can be both time-consuming and expensive. In this case, we are in the fortunate situation that atomicresolution HRTEM images can be reliably simulated, providing the training data at low cost. We have shown that it is possible to reliably identify the positions of the atoms in experimental micrographs of single sheets of defected graphene, and the atomic columns in micrographs of metallic nanoparticles, even when the signal-to-noise ratio is low due to the desire to limit beam exposure [3].

From a focal series of HRTEM images, it is possible to reconstruct the exit wave function. This can be used to extract further information about the atoms, such as the atomic species in two-dimensional materials, as shown recently for $\mathrm{MoS}_{2}$ [4]. A convolutional neural network can be trained to identify Mo and $\mathrm{S}$ atoms from a series of three HRTEM images with different defocus, thus bypassing the requirement of a full exit wave reconstruction (Figure 2).

Reducing the human component of image analysis reduces the risk that the preconceived biases of the researcher influence the analysis of the images. However, there is the risk that human bias is merely replaced by the bias of the neural network, typically reflecting the choices that were made when the training set was constructed. This can be addressed (but not completely eliminated) by generating multiple training sets using different rules and algorithms; using them to train multiple networks. Where the networks agree, the result can be considered reasonably robust whereas where they disagree care should be applied in interpreting the results.

In conclusion, we find that deep learning is a promising tool within electron microscopy, which may both accelerate analysis of large amounts of microscopy data, provide statistical data, and enable types of analysis that would not otherwise be possible, such as identifying chemical species in 2D materials. However, care must be taken that the neural network does not generate artifacts in the analysis by extrapolation from the specific training set. 


\section{References:}

[1] https://gitlab.com/schiotz/NeuralNetwork_HRTEM

[2] J Madsen et al., Adv. Theory Simul. 1 (2018), p. 1800037.

[3] J Madsen et al., Adv. Struct. Chem. Imag. 3 (2017), p. 14.

[4] C Kisielowski et al., Angew. Chem. Int. Ed. 49 (2010), p. 2708.

[5] AS Dreisig, B.Sc. thesis, Technical University of Denmark (2019).

[6] J Schiøtz et al., Microsc. Microanal. 24 (2018), p. 512.

A)

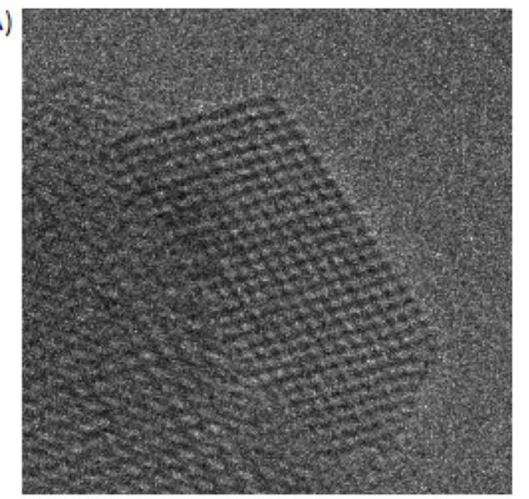

B)

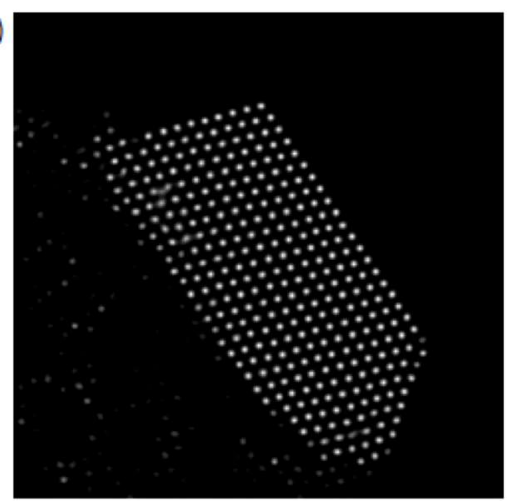

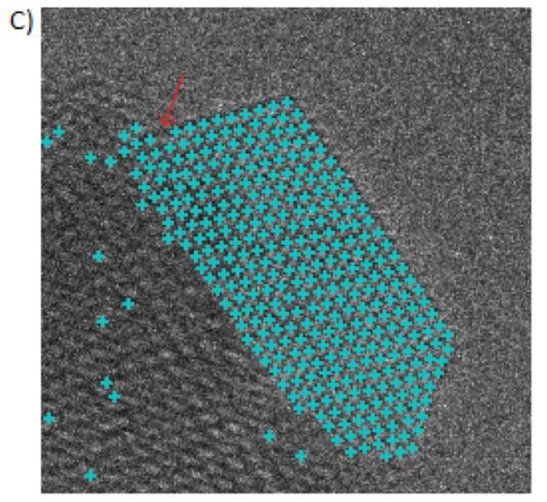

Figure 1. (A) Experimental HRTEM image of a gold nanoparticle on a ceria substrate in vaccum. (B) Output from the neural network. (C) Peak finding is used on the output from the neural network to identify the atoms. In spite of the contrast not being particularly strong in the upper right part of the image, the neural network locates the atoms with the exception of one (marked with a red arrow). Reproduced from [5].
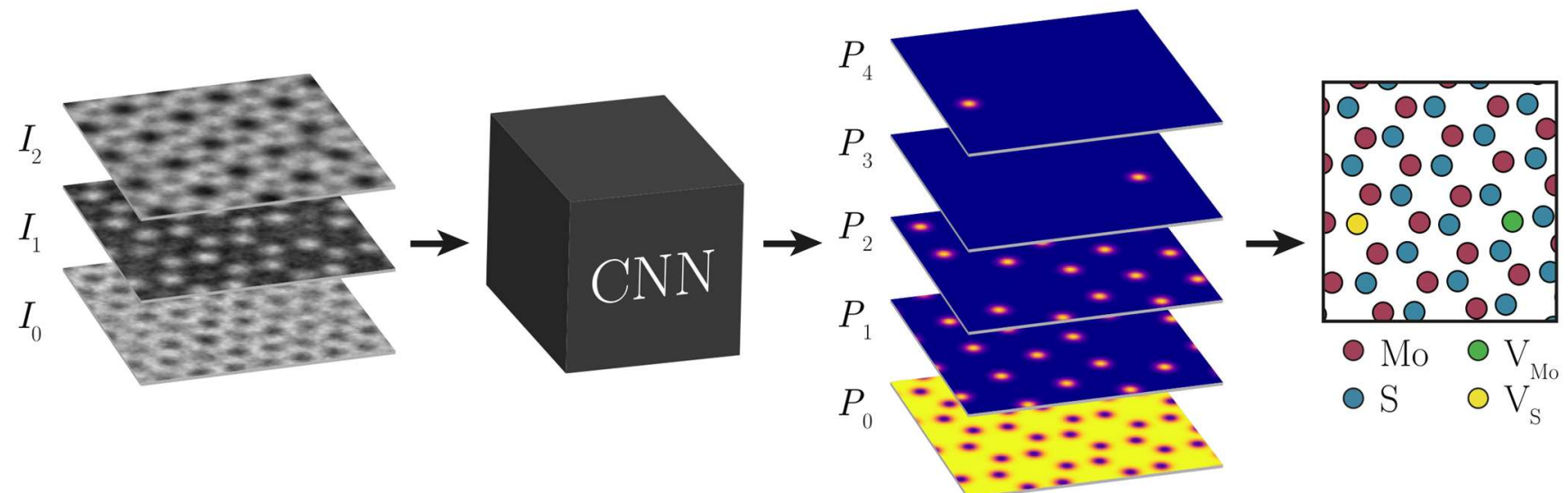

Figure 2. The neural net is fed three images of the same sample with different defocus. The output of the net is the probability of finding an "object" of a specific type at a given point. In this case, the set of possible objects are Mo atom, S atom, Mo vacancy, S vacancy and background. Reproduced from [6]. 